\title{
ON THE IDEALS OF ORLICZ TYPE OPERATORS
}

\author{
M. GuPtA AND L. R. ACHARYA
}

\begin{abstract}
We establish results on mappings of type $\ell_{M}$ defined by approximation numbers, for a given Orlicz function $M$. We associate the spaces of mappings of type $\ell_{M}$ with Köthe sequence spaces.
\end{abstract}

Mathematics subject classification (2010): 46A45, 47B06, 47L05.

Keywords and phrases: Approximation numbers, Orlicz function, sequence spaces.

\section{REFERENCES}

[1] B. Carl And I. Stephani, Entropy, compactness and the approximation of operators, Cambridge Univ. Press, Cambridge, 1990.

[2] P. K. Kamthan And M. Gupta, Sequence spaces and series, Marcel Dekker, Inc. New York, 1981.

[3] M. A. Krasnoselskit And Y. B. Rutitsky, Convex functions and Orlicz spaces, Groningen, Netherlands, 1961.

[4] J. Lindenstrauss And L. Tzafriri, Classical Banach Spaces I, Springer-Verlag, Berlin, Heidelberg, New York, 1977.

[5] A. Pietsch, Nuclear locally convex spaces, Springer-Verlag, Berlin, Heidelberg, New York, 1972.

[6] A. PietsCH, s-numbers of operators in Banach spaces, Studia Math. 51 (1974), pp. 201-223. 